\title{
New fractional-order multivalued problems with nonlocal nonlinear flux type integral boundary conditions
}

\author{
Bashir Ahmad ${ }^{1 *}$, Sotiris K Ntouyas ${ }^{1,2}$, Ahmed Alsaedi $^{1}$ and Faris Alzahrani ${ }^{1}$
}

\author{
"Correspondence: \\ bashirahmad_qau@yahoo.com \\ ${ }^{1}$ Nonlinear Analysis and Applied \\ Mathematics (NAAM)-Research \\ Group, Department of Mathematics, \\ Faculty of Science, King Abdulaziz \\ University, P.O. Box 80203, Jeddah, \\ 21589, Saudi Arabia \\ Full list of author information is \\ available at the end of the article
}

\begin{abstract}
In this paper, we study new fractional-order multivalued problems supplemented with nonlocal nonlinear flux type integral boundary conditions. Some existence results are obtained for convex as well as non-convex multivalued maps by applying standard fixed point theorems for such maps. We also discuss examples for the illustration of our results.
\end{abstract}

MSC: 34A60; 34A08; 34B15

Keywords: fractional differential inclusions; nonlocal conditions; integral boundary conditions; fixed point theorems

\section{Introduction}

In this paper, we investigate the existence of solutions for a fractional differential inclusion

$$
{ }^{c} D^{q} x(t) \in F(t, x(t)), \quad t \in[0,1], 1<q \leq 2,
$$

supplemented with nonlocal nonlinear flux type integral boundary conditions

$$
x(0)=\beta g\left(x^{\prime}(\eta)\right), \quad x^{\prime}(1)=\alpha \int_{0}^{\xi} x^{\prime}(s) d s, \quad 0<\xi, \eta<1,
$$

and

$$
x^{\prime}(0)=\alpha \int_{0}^{\xi} x^{\prime}(s) d s, \quad x(1)=\beta g\left(x^{\prime}(\eta)\right), \quad 0<\xi, \eta<1,
$$

where ${ }^{c} D^{q}$ denotes the Caputo fractional derivative of order $q, F:[0,1] \times \mathbb{R} \rightarrow \mathcal{P}(\mathbb{R})$ is a multivalued map, $\mathcal{P}(\mathbb{R})$ is the family of all nonempty subsets of $\mathbb{R}, g: \mathbb{R} \rightarrow \mathbb{R}$ is a continuous function and $\alpha, \beta$ are appropriate real constants with $\alpha \xi \neq 1$.

Fractional differential equations are found to be of great interest in view of their extensive applications in various scientific disciplines such as fluid mechanics, biomathematics, ecology, visco-elastodynamics, aerodynamics, control theory, electro-dynamics of complex medium, thermodynamics, electrical circuits, electron-analytical chemistry, etc. For details, we refer the reader to the books [1-6].

(c) 2015 Ahmad et al. This article is distributed under the terms of the Creative Commons Attribution 4.0 International License (http://creativecommons.org/licenses/by/4.0/), which permits unrestricted use, distribution, and reproduction in any medium, provided you give appropriate credit to the original author(s) and the source, provide a link to the Creative Commons license, and indicate if changes were made. 
The subject of boundary value problems has an enriched history, and several kinds of problems have been discussed over the years. In the past few years, fractional-order boundary value problems have received much attention, and the subject has been developed in an extensive manner. In fact it is a hot topic of research in mathematics and its applications. For some recent results on boundary value problems of fractional differential equations, we refer the reader to a series of papers [7-18] and the references cited therein.

Differential inclusions (multivalued differential equations), regarded as the generalization of single-valued differential equations, are found to be important mathematical modeling tools in certain problems of economics, optimal control, etc. and are widely studied by many authors; for instance, see [19-21] and the references therein. Examples and details of some recent works on differential inclusions of fractional order can be found in [22-28] and the references cited therein.

In this paper, we extend the study on fractional boundary value problems further by introducing a new class of problems of fractional differential inclusions supplemented with nonlocal nonlinear flux type integral boundary conditions. We obtain some existence results for the given problems for the cases of convex as well as non-convex multivalued maps. Our results are based on some standard theorems dealing with multivalued maps. The methods used here are well known, however their exposition in the context of problems (1.1)-(1.2) and (1.1)-(1.3) is new.

The paper is organized as follows. In Section 2 we present some related preliminary material from fractional calculus and multivalued analysis. We also describe the details of the proposed work. The main existence results for problem (1.1)-(1.2) are obtained in Section 3, and the examples illustrating the results of Section 3 are given in Section 4. We indicate the results for problem (1.1)-(1.3) in Section 5.

\section{Preliminaries}

This section is devoted to some preliminary concepts of fractional calculus and multivalued analysis. We also describe the work proposed in this paper together with the allied techniques.

Let us first recall some definitions of fractional calculus $[1,2]$ and prove an auxiliary result needed to define the solution for problem (1.1)-(1.2).

Definition 2.1 For an $(n-1)$-times absolutely continuous function $g:[0, \infty) \rightarrow \mathbb{R}$, the Caputo derivative of fractional order $q$ is defined as

$$
{ }^{c} D^{q} g(t)=\frac{1}{\Gamma(n-q)} \int_{0}^{t}(t-s)^{n-q-1} g^{(n)}(s) d s, \quad n-1<q<n, n=[q]+1,
$$

where $[q]$ denotes the integer part of the real number $q$.

Definition 2.2 The Riemann-Liouville fractional integral of order $q$ is defined as

$$
I^{q} g(t)=\frac{1}{\Gamma(q)} \int_{0}^{t} \frac{g(s)}{(t-s)^{1-q}} d s, \quad q>0
$$

provided the integral exists. 
Lemma 2.3 Let $y \in C([0,1], \mathbb{R})$ and $x \in C^{2}([0,1], \mathbb{R})$ be a solution of the linear boundary value problem

$$
\left\{\begin{array}{l}
{ }^{c} D^{q} x(t)=y(t), \quad t \in[0,1], 1<q \leq 2, \\
x(0)=\beta g\left(x^{\prime}(\eta)\right), \quad x^{\prime}(1)=\alpha \int_{0}^{\xi} x^{\prime}(s) d s, \quad 0 \leq \xi, \eta \leq 1 .
\end{array}\right.
$$

Then

$$
\begin{aligned}
x(t)= & \int_{0}^{t} \frac{(t-s)^{q-1}}{\Gamma(q)} y(s) d s \\
& +\frac{t}{1-\alpha \xi}\left(\alpha \int_{0}^{\xi} \int_{0}^{s} \frac{(s-\tau)^{q-2}}{\Gamma(q-1)} y(\tau) d \tau d s-\int_{0}^{1} \frac{(1-s)^{q-1}}{\Gamma(q)} y(s) d s\right) \\
& +\beta g\left(\int_{0}^{\eta} \frac{(\eta-s)^{q-2}}{\Gamma(q-1)} y(s) d s+\frac{\alpha}{1-\alpha \xi} \int_{0}^{\xi} \int_{0}^{s} \frac{(s-\tau)^{q-2}}{\Gamma(q-1)} y(\tau) d \tau d s\right. \\
& \left.-\frac{1}{1-\alpha \xi} \int_{0}^{1} \frac{(1-s)^{q-1}}{\Gamma(q)} y(s) d s\right) .
\end{aligned}
$$

Proof It is well known that the general solution of the fractional differential equation in (2.1) can be written as

$$
x(t)=c_{0}+c_{1} t+\int_{0}^{t} \frac{(t-s)^{q-1}}{\Gamma(q)} y(s) d s,
$$

where $c_{0}, c_{1} \in \mathbb{R}$ are arbitrary constants.

Applying the given boundary conditions, we find that

$$
c_{0}=\beta g\left(\int_{0}^{\eta} \frac{(\eta-s)^{q-2}}{\Gamma(q-1)} y(s) d s+c_{1}\right)
$$

and

$$
c_{1}=\frac{1}{1-\alpha \xi}\left(\alpha \int_{0}^{\xi} \int_{0}^{s} \frac{(s-\tau)^{q-2}}{\Gamma(q-1)} y(\tau) d \tau d s-\int_{0}^{1} \frac{(1-s)^{q-2}}{\Gamma(q-1)} y(s) d s\right) .
$$

Substituting the values of $c_{0}, c_{1}$ in (2.3), we get (2.2). This completes the proof.

Now we outline some background material on multivalued maps [29, 30].

For a normed space $(X,\|\cdot\|)$, let $\mathcal{P}_{c l}(X)=\{Y \in \mathcal{P}(X): Y$ is closed $\}, \mathcal{P}_{b}(X)=\{Y \in$ $\mathcal{P}(X): Y$ is bounded $\}, \mathcal{P}_{c p}(X)=\{Y \in \mathcal{P}(X): Y$ is compact $\}$, and $\mathcal{P}_{c p, c}(X)=\{Y \in \mathcal{P}(X)$ : $Y$ is compact and convex $\}$. A multivalued map $G: X \rightarrow \mathcal{P}(X)$ is convex (closed) valued if $G(x)$ is convex (closed) for all $x \in X$. The map $G$ is bounded on bounded sets if $G(\mathbb{B})=$ $\bigcup_{x \in \mathbb{B}} G(x)$ is bounded in $X$ for all $\mathbb{B} \in \mathcal{P}_{b}(X)$ (i.e., $\left.\sup _{x \in \mathbb{B}}\{\sup \{|y|: y \in G(x)\}\}<\infty\right)$. $G$ is called upper semicontinuous (u.s.c.) on $X$ if for each $x_{0} \in X$, the set $G\left(x_{0}\right)$ is a nonempty closed subset of $X$, and if for each open set $N$ of $X$ containing $G\left(x_{0}\right)$, there exists an open neighborhood $\mathcal{N}_{0}$ of $x_{0}$ such that $G\left(\mathcal{N}_{0}\right) \subseteq N$. G is said to be completely continuous if $G(\mathbb{B})$ is relatively compact for every $\mathbb{B} \in \mathcal{P}_{b}(X)$. If the multivalued map $G$ is completely continuous with nonempty compact values, then $G$ is u.s.c. if and only if $G$ has a closed graph, i.e., $x_{n} \rightarrow x_{*}, y_{n} \rightarrow y_{*}, y_{n} \in G\left(x_{n}\right)$ imply $y_{*} \in G\left(x_{*}\right)$. $G$ has a fixed point if there is $x \in X$ such that $x \in G(x)$. The fixed point set of the multivalued operator $G$ will be denoted by Fix $G$. A multivalued map $G:[0,1] \rightarrow \mathcal{P}_{c l}(\mathbb{R})$ is said to be measurable if for every $y \in \mathbb{R}$, 
the function

$$
t \longmapsto d(y, G(t))=\inf \{|y-z|: z \in G(t)\}
$$

is measurable.

For each $y \in C([0,1], \mathbb{R})$, define the set of selections of $F$ by

$$
S_{F, y}:=\left\{v \in L^{1}([0,1], \mathbb{R}): v(t) \in F(t, y(t)) \text { for a.e. } t \in[0,1]\right\} .
$$

Next we characterize the proposed work and provide the necessary details for achieving the main results.

To discuss the existence of solutions for problem (1.1)-(1.2) with a nonconvex valued right-hand side (the Lipschitz case), we need the following auxiliary material.

Let $(X, d)$ be a metric space induced from the normed space $(X ;\|\cdot\|)$. Consider $H_{d}$ : $\mathcal{P}(X) \times \mathcal{P}(X) \rightarrow \mathbb{R} \cup\{\infty\}$ given by

$$
H_{d}(A, B)=\max \left\{\sup _{a \in A} d(a, B), \sup _{b \in B} d(A, b)\right\},
$$

where $d(A, b)=\inf _{a \in A} d(a ; b)$ and $d(a, B)=\inf _{b \in B} d(a ; b)$. Then $\left(\mathcal{P}_{b, c l}(X), H_{d}\right)$ is a metric space and $\left(\mathcal{P}_{c l}(X), H_{d}\right)$ is a generalized metric space (see [31]).

Definition 2.4 A multivalued operator $N: X \rightarrow \mathcal{P}_{c l}(X)$ is called:

(a) $\gamma$-Lipschitz if and only if there exists $\gamma>0$ such that

$$
H_{d}(N(x), N(y)) \leq \gamma d(x, y) \quad \text { for each } x, y \in X
$$

(b) a contraction if and only if it is $\gamma$-Lipschitz with $\gamma<1$.

Lemma 2.5 ([32]) Let $(X, d)$ be a complete metric space. If $N: X \rightarrow \mathcal{P}_{c l}(X)$ is a contraction, then Fix $N \neq \emptyset$.

To establish the existence result when the multivalued map $F$ in (1.1) is convex-valued (the upper semicontinuous case), we recall the following definitions and known results.

Definition 2.6 A multivalued map $F:[0,1] \times \mathbb{R} \rightarrow \mathcal{P}(\mathbb{R})$ is said to be Carathéodory if

(i) $t \longmapsto F(t, x)$ is measurable for each $x \in \mathbb{R}$;

(ii) $x \longmapsto F(t, x)$ is upper semicontinuous for almost all $t \in[0,1]$;

Further a Carathéodory function $F$ is called $L^{1}$-Carathéodory if

(iii) for each $\rho>0$, there exists $\varphi_{\rho} \in L^{1}\left([0,1], \mathbb{R}^{+}\right)$such that

$$
\|F(t, x)\|=\sup \{|v|: v \in F(t, x)\} \leq \varphi_{\rho}(t)
$$

for all $\|x\| \leq \rho$ and for a.e. $t \in[0,1]$.

We define the graph of a multivalued map $G$ to be the set $\operatorname{Gr}(G)=\{(x, y) \in X \times Y, y \in$ $G(x)\}$ and recall two results for closed graphs and upper-semicontinuity.

Lemma 2.7 ([29], Proposition 1.2) If $G: X \rightarrow \mathcal{P}_{c l}(Y)$ is u.s.c., then $\operatorname{Gr}(G)$ is a closed subset of $X \times Y$; i.e., for every sequence $\left\{x_{n}\right\}_{n \in \mathbb{N}} \subset X$ and $\left\{y_{n}\right\}_{n \in \mathbb{N}} \subset Y$, if when $n \rightarrow \infty, x_{n} \rightarrow x_{*}$, 
$y_{n} \rightarrow y_{*}$ and $y_{n} \in G\left(x_{n}\right)$, then $y_{*} \in G\left(x_{*}\right)$. Conversely, if $G$ is completely continuous and has a closed graph, then it is upper semicontinuous.

Lemma 2.8 ([33]) Let $X$ be a Banach space. Let $F:[0,1] \times X \rightarrow \mathcal{P}_{c p, c}(X)$ be an $L^{1}$ Carathéodory multivalued map, and let $\Theta$ be a linear continuous mapping from $L^{1}([0,1], X)$ to $C([0,1], X)$. Then the operator

$$
\Theta \circ S_{F}: C([0,1], X) \rightarrow \mathcal{P}_{c p, c}(C([0,1], X)), \quad x \longmapsto\left(\Theta \circ S_{F}\right)(x)=\Theta\left(S_{F, x}\right)
$$

is a closed graph operator in $C([0,1], X) \times C([0,1], X)$.

Lemma 2.9 (Nonlinear alternative for Kakutani maps) [34] Let E be a Banach space, C be a closed convex subset of $E, U$ be an open subset of $C$ and $0 \in U$. Suppose that $F: \bar{U} \rightarrow$ $\mathcal{P}_{c p, c}(C)$ is an upper semicontinuous compact map. Then either

(i) F has a fixed point in $\bar{U}$, or

(ii) there is $u \in \partial U$ and $\lambda \in(0,1)$ with $u \in \lambda F(u)$.

Finally, we study the case when the multivalued map $F$ in (1.1) is not necessarily convexvalued (the lower semicontinuous case). In this case, the background material is outlined as follows.

Let $X$ be a nonempty closed subset of a Banach space $E$ and $G: X \rightarrow \mathcal{P}(E)$ be a multivalued operator with nonempty closed values. $G$ is lower semicontinuous (l.s.c.) if the set $\{y \in X: G(y) \cap B \neq \emptyset\}$ is open for any open set $B$ in $E$. Let $A$ be a subset of $[0,1] \times \mathbb{R}$. $A$ is $\mathcal{L} \otimes \mathcal{B}$ measurable if $A$ belongs to the $\sigma$-algebra generated by all sets of the form $\mathcal{J} \times \mathcal{D}$, where $\mathcal{J}$ is Lebesgue measurable in $[0,1]$ and $\mathcal{D}$ is Borel measurable in $\mathbb{R}$. A subset $\mathcal{A}$ of $L^{1}([0,1], \mathbb{R})$ is decomposable if for all $u, v \in \mathcal{A}$ and measurable $\mathcal{J} \subset[0,1]=J$, the function $u \chi_{\mathcal{J}}+v \chi_{\mathcal{J}-\mathcal{J}} \in \mathcal{A}$, where $\chi_{\mathcal{J}}$ stands for the characteristic function of $\mathcal{J}$.

Definition 2.10 Let $Y$ be a separable metric space and let $N: Y \rightarrow \mathcal{P}\left(L^{1}([0,1], \mathbb{R})\right)$ be a multivalued operator. We say $N$ has a property $(\mathrm{BC})$ if $N$ is lower semicontinuous (l.s.c.) and has nonempty closed and decomposable values.

Let $F:[0,1] \times \mathbb{R} \rightarrow \mathcal{P}(\mathbb{R})$ be a multivalued map with nonempty compact values. Define a multivalued operator $\mathcal{F}: C([0,1] \times \mathbb{R}) \rightarrow \mathcal{P}\left(L^{1}([0,1], \mathbb{R})\right)$ associated with $F$ as

$$
\mathcal{F}(x)=\left\{w \in L^{1}([0,1], \mathbb{R}): w(t) \in F(t, x(t)) \text { for a.e. } t \in[0,1]\right\},
$$

which is called the Nemytskii operator associated with $F$.

Definition 2.11 Let $F:[0,1] \times \mathbb{R} \rightarrow \mathcal{P}(\mathbb{R})$ be a multivalued function with nonempty compact values. We say $F$ is of lower semicontinuous type (l.s.c. type) if its associated Nemytskii operator $\mathcal{F}$ is lower semicontinuous and has nonempty closed and decomposable values.

Lemma 2.12 ([35]) Let $Y$ be a separable metric space and let $N: Y \rightarrow \mathcal{P}\left(L^{1}([0,1], \mathbb{R})\right)$ be a multivalued operator satisfying the property $(B C)$. Then $N$ has a continuous selection, that is, there exists a continuous function (single-valued) $g: Y \rightarrow L^{1}([0,1], \mathbb{R})$ such that $g(x) \in N(x)$ for every $x \in Y$. 


\section{Existence results}

Definition 3.1 A function $x \in C^{2}([0,1], \mathbb{R})$ is said to be a solution of the boundary value problem (1.1)-(1.2) if $x(0)=\beta g\left(x^{\prime}(\eta)\right), x^{\prime}(1)=\alpha \int_{0}^{\xi} x^{\prime}(s) d s$, and there exists a function $v \in$ $S_{F, x}$ such that

$$
\begin{aligned}
x(t)= & \int_{0}^{t} \frac{(t-s)^{q-1}}{\Gamma(q)} v(s) d s \\
& +\frac{t}{1-\alpha \xi}\left(\alpha \int_{0}^{\xi} \int_{0}^{s} \frac{(s-\tau)^{q-2}}{\Gamma(q-1)} v(\tau) d \tau d s-\int_{0}^{1} \frac{(1-s)^{q-1}}{\Gamma(q)} v(s) d s\right) \\
& +\beta g\left(\int_{0}^{\eta} \frac{(\eta-s)^{q-2}}{\Gamma(q-1)} v(s) d s+\frac{\alpha}{1-\alpha \xi} \int_{0}^{\xi} \int_{0}^{s} \frac{(s-\tau)^{q-2}}{\Gamma(q-1)} v(\tau) d \tau d s\right. \\
& \left.-\frac{1}{1-\alpha \xi} \int_{0}^{1} \frac{(1-s)^{q-1}}{\Gamma(q)} v(s) d s\right), \quad t \in[0,1] .
\end{aligned}
$$

For the sake of convenience, we set

$$
\Lambda=\frac{1}{\Gamma(q+1)}\left(1+|\beta| q \eta^{q-1}+\frac{|\alpha| \xi^{q}+1}{|1-\alpha \xi|}(1+|\beta|)\right)
$$

Now we are in a position to present our first existence result for problem (1.1)-(1.2) which deals with a nonconvex valued right-hand side of (1.1).

Theorem 3.2 (The Lipschitz case) Assume that

$\left(\mathrm{A}_{1}\right) F:[0,1] \times \mathbb{R} \rightarrow \mathcal{P}_{c p}(\mathbb{R})$ is such that $F(\cdot, x):[0,1] \rightarrow \mathcal{P}_{c p}(\mathbb{R})$ is measurable for each $x \in \mathbb{R}$

$\left(\mathrm{A}_{2}\right) H_{d}(F(t, x), F(t, \bar{x})) \leq m(t)|x-\bar{x}|$ for almost all $t \in[0,1]$ and $x, \bar{x} \in \mathbb{R}$ with $m \in$ $C\left([0,1], \mathbb{R}^{+}\right)$and $d(0, F(t, 0)) \leq m(t)$ for almost all $t \in[0,1]$;

$\left(\mathrm{A}_{3}\right) g: \mathbb{R} \rightarrow \mathbb{R}$ is continuous and $|g(v)| \leq|v|, \forall v \in \mathbb{R}$.

Then the boundary value problem (1.1)-(1.2) has at least one solution on $[0,1]$ if $\|m\| \Lambda<1$, where $\Lambda$ is given by (3.2).

Proof Define the operator $\Omega_{F}: C([0,1], \mathbb{R}) \rightarrow \mathcal{P}(C([0,1], \mathbb{R}))$ by

$$
\Omega_{F}(x)=\left\{\begin{array}{l}
h \in C([0,1], \mathbb{R}): \\
h(t)=\left\{\begin{array}{l}
\int_{0}^{t} \frac{(t-s)^{q-1}}{\Gamma(q)} v(s) d s \\
\quad+\frac{t}{1-\alpha \xi}\left(\alpha \int_{0}^{\xi} \int_{0}^{s} \frac{(s-\tau)^{q-2}}{\Gamma(q-1)} v(\tau) d \tau d s-\int_{0}^{1} \frac{(1-s)^{q-1}}{\Gamma(q)} v(s) d s\right) \\
+\beta g\left(\int_{0}^{\eta} \frac{(\eta-s)^{q-2}}{\Gamma(q-1)} v(s) d s+\frac{\alpha}{1-\alpha \xi} \int_{0}^{\xi} \int_{0}^{s} \frac{(s-\tau)^{q-2}}{\Gamma(q-1)} v(\tau) d \tau d s\right. \\
\left.-\frac{1}{1-\alpha \xi} \int_{0}^{1} \frac{(1-s)^{q-1}}{\Gamma(q)} v(s) d s\right)
\end{array}\right\}
\end{array}\right.
$$

for $v \in S_{F, x}$.

Observe that the set $S_{F, x}$ is nonempty for each $x \in C([0,1], \mathbb{R})$ by the assumption $\left(\mathrm{A}_{1}\right)$, so $F$ has a measurable selection (see Theorem III.6 [36]). Now we show that the operator $\Omega_{F}$ satisfies the assumptions of Lemma 2.5 . To show that $\left.\Omega_{F}(x) \in \mathcal{P}_{c l}(C[0,1], \mathbb{R})\right)$ for each $x \in C([0,1], \mathbb{R})$, let $\left\{u_{n}\right\}_{n \geq 0} \in \Omega_{F}(x)$ be such that $u_{n} \rightarrow u(n \rightarrow \infty)$ in $C([0,1], \mathbb{R})$. Then $u \in C([0,1], \mathbb{R})$ and there exists $v_{n} \in S_{F, x_{n}}$ such that, for each $t \in[0,1]$, 


$$
\begin{aligned}
u_{n}(t)= & \int_{0}^{t} \frac{(t-s)^{q-1}}{\Gamma(q)} v_{n}(s) d s \\
& +\frac{t}{1-\alpha \xi}\left(\alpha \int_{0}^{\xi} \int_{0}^{s} \frac{(s-\tau)^{q-2}}{\Gamma(q-1)} v_{n}(\tau) d \tau d s-\int_{0}^{1} \frac{(1-s)^{q-1}}{\Gamma(q)} v_{n}(s) d s\right) \\
& +\beta g\left(\int_{0}^{\eta} \frac{(\eta-s)^{q-2}}{\Gamma(q-1)} v_{n}(s) d s+\frac{\alpha}{1-\alpha \xi} \int_{0}^{\xi} \int_{0}^{s} \frac{(s-\tau)^{q-2}}{\Gamma(q-1)} v_{n}(\tau) d \tau d s\right. \\
& \left.-\frac{1}{1-\alpha \xi} \int_{0}^{1} \frac{(1-s)^{q-1}}{\Gamma(q)} v_{n}(s) d s\right) .
\end{aligned}
$$

As $F$ has compact values, we pass onto a subsequence (if necessary) to obtain that $v_{n}$ converges to $v$ in $L^{1}([0,1], \mathbb{R})$. Thus, $v \in S_{F, x}$ and for each $t \in[0,1]$, we have

$$
\begin{aligned}
v_{n}(t) \rightarrow & v(t)=\int_{0}^{t} \frac{(t-s)^{q-1}}{\Gamma(q)} v(s) d s \\
& +\frac{t}{1-\alpha \xi}\left(\alpha \int_{0}^{\xi} \int_{0}^{s} \frac{(s-\tau)^{q-2}}{\Gamma(q-1)} v(\tau) d \tau d s-\int_{0}^{1} \frac{(1-s)^{q-1}}{\Gamma(q)} v(s) d s\right) \\
& +\beta g\left(\int_{0}^{\eta} \frac{(\eta-s)^{q-2}}{\Gamma(q-1)} v(s) d s+\frac{\alpha}{1-\alpha \xi} \int_{0}^{\xi} \int_{0}^{s} \frac{(s-\tau)^{q-2}}{\Gamma(q-1)} v(\tau) d \tau d s\right. \\
& \left.-\frac{1}{1-\alpha \xi} \int_{0}^{1} \frac{(1-s)^{q-1}}{\Gamma(q)} v(s) d s\right) .
\end{aligned}
$$

Hence, $u \in \Omega_{F}(x)$.

Next we show that there exists $\delta<1$ such that

$$
H_{d}\left(\Omega_{F}(x), \Omega_{F}(\bar{x})\right) \leq \delta\|x-\bar{x}\| \quad \text { for each } x, \bar{x} \in C^{2}([0,1], \mathbb{R}) .
$$

Let $x, \bar{x} \in C^{2}([0,1], \mathbb{R})$ and $h_{1} \in \Omega_{F}(x)$. Then there exists $v_{1}(t) \in F(t, x(t))$ such that, for each $t \in[0,1]$,

$$
\begin{aligned}
h_{1}(t)= & \int_{0}^{t} \frac{(t-s)^{q-1}}{\Gamma(q)} v_{1}(s) d s \\
& +\frac{t}{1-\alpha \xi}\left(\alpha \int_{0}^{\xi} \int_{0}^{s} \frac{(s-\tau)^{q-2}}{\Gamma(q-1)} v_{1}(\tau) d \tau d s-\int_{0}^{1} \frac{(1-s)^{q-1}}{\Gamma(q)} v_{1}(s) d s\right) \\
& +\beta g\left(\int_{0}^{\eta} \frac{(\eta-s)^{q-2}}{\Gamma(q-1)} v_{1}(s) d s+\frac{\alpha}{1-\alpha \xi} \int_{0}^{\xi} \int_{0}^{s} \frac{(s-\tau)^{q-2}}{\Gamma(q-1)} v_{1}(\tau) d \tau d s\right. \\
& \left.-\frac{1}{1-\alpha \xi} \int_{0}^{1} \frac{(1-s)^{q-1}}{\Gamma(q)} v_{1}(s) d s\right) .
\end{aligned}
$$

By $\left(\mathrm{A}_{2}\right)$, we have

$$
H_{d}(F(t, x), F(t, \bar{x})) \leq m(t)|x(t)-\bar{x}(t)| .
$$

So, there exists $w \in F(t, \bar{x}(t))$ such that

$$
\left|v_{1}(t)-w\right| \leq m(t)|x(t)-\bar{x}(t)|, \quad t \in[0,1] .
$$

Define $U:[0,1] \rightarrow \mathcal{P}(\mathbb{R})$ by

$$
U(t)=\left\{w \in \mathbb{R}:\left|v_{1}(t)-w\right| \leq m(t)|x(t)-\bar{x}(t)|\right\} .
$$


Since the multivalued operator $U(t) \cap F(t, \bar{x}(t))$ is measurable (Proposition III.4 [36]), there exists a function $v_{2}(t)$ which is a measurable selection for $U$. So $v_{2}(t) \in F(t, \bar{x}(t))$ and for each $t \in[0,1]$, we have $\left|v_{1}(t)-v_{2}(t)\right| \leq m(t)|x(t)-\bar{x}(t)|$.

For each $t \in[0,1]$, let us define

$$
\begin{aligned}
h_{2}(t)= & \int_{0}^{t} \frac{(t-s)^{q-1}}{\Gamma(q)} v_{2}(s) d s \\
& +\frac{t}{1-\alpha \xi}\left(\alpha \int_{0}^{\xi} \int_{0}^{s} \frac{(s-\tau)^{q-2}}{\Gamma(q-1)} v_{2}(\tau) d \tau d s-\int_{0}^{1} \frac{(1-s)^{q-1}}{\Gamma(q)} v_{2}(s) d s\right) \\
& +\beta g\left(\int_{0}^{\eta} \frac{(\eta-s)^{q-2}}{\Gamma(q-1)} v_{2}(s) d s+\frac{\alpha}{1-\alpha \xi} \int_{0}^{\xi} \int_{0}^{s} \frac{(s-\tau)^{q-2}}{\Gamma(q-1)} v_{2}(\tau) d \tau d s\right. \\
& \left.-\frac{1}{1-\alpha \xi} \int_{0}^{1} \frac{(1-s)^{q-1}}{\Gamma(q)} v_{2}(s) d s\right) .
\end{aligned}
$$

Thus,

$$
\begin{aligned}
\left|h_{1}(t)-h_{2}(t)\right| \leq & \int_{0}^{t} \frac{(t-s)^{q-1}}{\Gamma(q)}\left|v_{1}(s)-v_{2}(s)\right| d s \\
& +\frac{t}{|1-\alpha \xi|}\left(|\alpha| \int_{0}^{\xi} \int_{0}^{s} \frac{(s-\tau)^{q-2}}{\Gamma(q-1)}\left|v_{1}(\tau)-v_{2}(\tau)\right| d \tau d s\right. \\
& \left.+\int_{0}^{1} \frac{(1-s)^{q-1}}{\Gamma(q)}\left|v_{1}(s)-v_{2}(s)\right| d s\right) \\
& +|\beta| g\left(\int_{0}^{\eta} \frac{(\eta-s)^{q-2}}{\Gamma(q-1)}\left|v_{1}(s)-v_{2}(s)\right|(s) d s\right. \\
& +\frac{|\alpha|}{|1-\alpha \xi|} \int_{0}^{\xi} \int_{0}^{s} \frac{(s-\tau)^{q-2}}{\Gamma(q-1)}\left|v_{1}(\tau)-v_{2}(\tau)\right| d \tau d s \\
& \left.+\frac{1}{|1-\alpha \xi|} \int_{0}^{1} \frac{(1-s)^{q-1}}{\Gamma(q)}\left|v_{1}(s)-v_{2}(s)\right|(s) d s\right) \\
\leq & \|m\|\left\{\frac{1}{\Gamma(q+1)}+\frac{1}{|1-\alpha \xi|}\left(\frac{|\alpha| \xi^{q}}{\Gamma(q+1)}+\frac{1}{\Gamma(q+1)}\right)\right. \\
& \left.+|\beta|\left(\frac{\eta^{q-1}}{\Gamma(q)}+\frac{|\alpha|}{|1-\alpha \xi|} \frac{\xi^{q}}{\Gamma(q+1)}+\frac{1}{|1-\alpha \xi|} \frac{1}{\Gamma(q+1)}\right)\right\}\|x-\bar{x}\| \\
= & \|m\| \Lambda\|x-\bar{x}\| .
\end{aligned}
$$

Hence,

$$
\left\|h_{1}-h_{2}\right\| \leq\|m\| \Lambda\|x-\bar{x}\| .
$$

Analogously, interchanging the roles of $x$ and $\bar{x}$, we obtain

$$
H_{d}\left(\Omega_{F}(x), \Omega_{F}(\bar{x})\right) \leq\|m\| \Lambda\|x-\bar{x}\|
$$

where $\delta=\|m\| \Lambda<1$. So $\Omega_{F}$ is a contraction. Hence it follows by Lemma 2.5 that $\Omega_{F}$ has a fixed point $x$ which is a solution of (1.1)-(1.2). This completes the proof. 
Our next result concerns the existence of the solutions for problem (1.1)-(1.2) with a convex-valued multivalued map.

Theorem 3.3 (The upper semicontinuous case) Assume that $\left(\mathrm{A}_{3}\right)$ holds. In addition we suppose that

$\left(\mathrm{H}_{1}\right) \quad F:[0,1] \times \mathbb{R} \rightarrow \mathcal{P}(\mathbb{R})$ is Carathéodory and has nonempty compact and convex values;

$\left(\mathrm{H}_{2}\right)$ there exists a continuous nondecreasing function $\psi:[0, \infty) \rightarrow(0, \infty)$ and a function $\phi \in C\left([0,1], \mathbb{R}^{+}\right)$such that

$$
\|F(t, x)\|_{\mathcal{P}}:=\sup \{|y|: y \in F(t, x)\} \leq \phi(t) \psi(\|x\|) \quad \text { for each }(t, x) \in[0,1] \times \mathbb{R}
$$

$\left(\mathrm{H}_{3}\right)$ there exists a constant $M>0$ such that

$$
\frac{M}{\psi(M)\|\phi\| \Lambda}>1
$$

where $\Lambda$ is defined by (3.2).

Then the boundary value problem (1.1)-(1.2) has at least one solution on $[0,1]$.

Proof Consider the operator $\Omega_{F}: C([0,1], \mathbb{R}) \rightarrow \mathcal{P}(C([0,1], \mathbb{R}))$ defined in the beginning of the proof of Theorem 3.2. We will show that $\Omega_{F}$ satisfies the assumptions of the nonlinear alternative of Leray-Schauder type. The proof consists of several steps. As the first step, we show that $\Omega_{F}$ is convex for each $x \in C([0,1], \mathbb{R})$. This step is obvious since $S_{F, x}$ is convex ( $F$ has convex values), and therefore we omit the proof.

In the second step, we show that $\Omega_{F}$ maps bounded sets (balls) into bounded sets in $C([0,1], \mathbb{R})$. For a positive number $\rho$, let $B_{\rho}=\{x \in C([0,1], \mathbb{R}):\|x\| \leq \rho\}$ be a bounded ball in $C([0,1], \mathbb{R})$. Then, for each $h \in \Omega_{F}(x), x \in B_{\rho}$, there exists $v \in S_{F, x}$ such that

$$
\begin{aligned}
h(t)= & \int_{0}^{t} \frac{(t-s)^{q-1}}{\Gamma(q)} v(s) d s \\
& +\frac{t}{1-\alpha \xi}\left(\alpha \int_{0}^{\xi} \int_{0}^{s} \frac{(s-\tau)^{q-2}}{\Gamma(q-1)} v(\tau) d \tau d s-\int_{0}^{1} \frac{(1-s)^{q-1}}{\Gamma(q)} v(s) d s\right) \\
& +\beta g\left(\int_{0}^{\eta} \frac{(\eta-s)^{q-2}}{\Gamma(q-1)} v(s) d s+\frac{\alpha}{1-\alpha \xi} \int_{0}^{\xi} \int_{0}^{s} \frac{(s-\tau)^{q-2}}{\Gamma(q-1)} v(\tau) d \tau d s\right. \\
& \left.-\frac{1}{1-\alpha \xi} \int_{0}^{1} \frac{(1-s)^{q-1}}{\Gamma(q)} v(s) d s\right), \quad t \in[0,1] .
\end{aligned}
$$

Then for $t \in[0,1]$ we have

$$
\begin{aligned}
|h(t)| \leq & \int_{0}^{t} \frac{(t-s)^{q-1}}{\Gamma(q)}|v(s)| d s \\
& +\frac{t}{|1-\alpha \xi|}\left(|\alpha| \int_{0}^{\xi} \int_{0}^{s} \frac{(s-\tau)^{q-2}}{\Gamma(q-1)}|v(\tau)| d \tau d s+\int_{0}^{1} \frac{(1-s)^{q-1}}{\Gamma(q)}|v(s)| d s\right) \\
& +|\beta| g\left(\int_{0}^{\eta} \frac{(\eta-s)^{q-2}}{\Gamma(q-1)}|v(s)| d s+\frac{|\alpha|}{|1-\alpha \xi|} \int_{0}^{\xi} \int_{0}^{s} \frac{(s-\tau)^{q-2}}{\Gamma(q-1)}|v(\tau)| d \tau d s\right.
\end{aligned}
$$




$$
\begin{aligned}
& \left.+\frac{1}{|1-\alpha \xi|} \int_{0}^{1} \frac{(1-s)^{q-1}}{\Gamma(q)}|v(s)| d s\right) \\
\leq & \psi(\|x\|)\|\phi\|\left\{\frac{1}{\Gamma(q+1)}+\frac{1}{|1-\alpha \xi|}\left(\frac{|\alpha| \xi^{q}}{\Gamma(q+1)}+\frac{1}{\Gamma(q+1)}\right)\right. \\
& \left.+|\beta|\left(\frac{\eta^{q-1}}{\Gamma(q)}+\frac{|\alpha|}{|1-\alpha \xi|} \frac{\xi^{q}}{\Gamma(q+1)}+\frac{1}{|1-\alpha \xi|} \frac{1}{\Gamma(q+1)}\right)\right\} \\
= & \psi(\|x\|)\|\phi\| \Lambda .
\end{aligned}
$$

\section{Consequently,}

$$
\|h\| \leq \psi(\rho)\|\phi\| \Lambda
$$

Now we show that $\Omega_{F}$ maps bounded sets into equicontinuous sets of $C([0,1], \mathbb{R})$. Let $t_{1}, t_{2} \in[0,1]$ with $t_{1}<t_{2}$ and $x \in B_{\rho}$. For each $h \in \Omega_{F}(x)$, we obtain

$$
\begin{aligned}
\mid h\left(t_{2}\right) & -h\left(t_{1}\right) \mid \\
\leq & \left|\frac{1}{\Gamma(\alpha)} \int_{0}^{t_{2}}\left(t_{2}-s\right)^{q-1} v(s) d s-\frac{1}{\Gamma(\alpha)} \int_{0}^{t_{1}}\left(t_{1}-s\right)^{q-1} v(s) d s\right| \\
& +\frac{\left|t_{2}-t_{1}\right|}{|1-\alpha \xi|}\left(|\alpha| \int_{0}^{\xi} \int_{0}^{s} \frac{(s-\tau)^{q-2}}{\Gamma(q-1)}|v(\tau)| d \tau d s+\int_{0}^{1} \frac{(1-s)^{q-1}}{\Gamma(q)}|v(s)| d s\right) \\
\leq & \frac{1}{\Gamma(\alpha)} \int_{0}^{t_{1}}\left[\left(t_{2}-s\right)^{q-1}-\left(t_{1}-s\right)^{q-1}\right] \phi(s) \psi(r) d s+\frac{1}{\Gamma(\alpha)} \int_{t_{1}}^{t_{2}}\left(t_{2}-s\right)^{q-1} \phi(s) \psi(r) d s \\
& +\frac{\left|t_{2}-t_{1}\right|}{|1-\alpha \xi|}\left(|\alpha| \int_{0}^{\xi} \int_{0}^{s} \frac{(s-\tau)^{q-2}}{\Gamma(q-1)} \phi(s) \psi(r) d \tau d s+\int_{0}^{1} \frac{(1-s)^{q-1}}{\Gamma(q)} \phi(s) \psi(r) d s\right) \\
\leq & \frac{\|\phi\| \psi(\rho)}{\Gamma(\alpha)} \int_{0}^{t_{1}}\left[\left(t_{2}-s\right)^{q-1}-\left(t_{1}-s\right)^{q-1}\right] d s+\frac{\|\phi\| \psi(\rho)}{\Gamma(\alpha)} \int_{t_{1}}^{t_{2}}\left(t_{2}-s\right)^{q-1} d s \\
& +\frac{\|\phi\| \psi(\rho)\left|t_{2}-t_{1}\right|}{|1-\alpha \xi|}\left(\frac{|\alpha| \xi \xi^{q}}{\Gamma(q+1)}+\frac{1}{\Gamma(q+1)}\right) .
\end{aligned}
$$

Obviously the right-hand side of the above inequality tends to zero independently of $x \in$ $B_{\rho}$ as $t_{2}-t_{1} \rightarrow 0$. As $\Omega_{F}$ satisfies the above three assumptions, therefore it follows by the Ascoli-Arzelà theorem that $\Omega_{F}: C([0,1], \mathbb{R}) \rightarrow \mathcal{P}(C([0,1], \mathbb{R}))$ is completely continuous.

In our next step, we show that $\Omega_{F}$ is upper semicontinuous. To this end it is sufficient to show that $\Omega_{F}$ has a closed graph by Lemma 2.7. Let $x_{n} \rightarrow x_{*}, h_{n} \in \Omega_{F}\left(x_{n}\right)$ and $h_{n} \rightarrow h_{*}$. Then we need to show that $h_{*} \in \Omega_{F}\left(x_{*}\right)$. Associated with $h_{n} \in \Omega_{F}\left(x_{n}\right)$, there exists $v_{n} \in S_{F, x_{n}}$ such that for each $t \in[0,1]$,

$$
\begin{aligned}
h_{n}(t)= & \int_{0}^{t} \frac{(t-s)^{q-1}}{\Gamma(q)} v_{n}(s) d s \\
& +\frac{t}{1-\alpha \xi}\left(\alpha \int_{0}^{\xi} \int_{0}^{s} \frac{(s-\tau)^{q-2}}{\Gamma(q-1)} v_{n}(\tau) d \tau d s-\int_{0}^{1} \frac{(1-s)^{q-1}}{\Gamma(q)} v_{n}(s) d s\right) \\
& +\beta g\left(\int_{0}^{\eta} \frac{(\eta-s)^{q-2}}{\Gamma(q-1)} v_{n}(s) d s+\frac{\alpha}{1-\alpha \xi} \int_{0}^{\xi} \int_{0}^{s} \frac{(s-\tau)^{q-2}}{\Gamma(q-1)} v_{n}(\tau) d \tau d s\right. \\
& \left.-\frac{1}{1-\alpha \xi} \int_{0}^{1} \frac{(1-s)^{q-1}}{\Gamma(q)} v_{n}(s) d s\right) .
\end{aligned}
$$


Thus it suffices to show that there exists $v_{*} \in S_{F, x_{*}}$ such that for each $t \in[0,1]$,

$$
\begin{aligned}
h_{*}(t)= & \int_{0}^{t} \frac{(t-s)^{q-1}}{\Gamma(q)} v_{*}(s) d s \\
& +\frac{t}{1-\alpha \xi}\left(\alpha \int_{0}^{\xi} \int_{0}^{s} \frac{(s-\tau)^{q-2}}{\Gamma(q-1)} v_{*}(\tau) d \tau d s-\int_{0}^{1} \frac{(1-s)^{q-1}}{\Gamma(q)} v_{*}(s) d s\right) \\
& +\beta g\left(\int_{0}^{\eta} \frac{(\eta-s)^{q-2}}{\Gamma(q-1)} v_{*}(s) d s+\frac{\alpha}{1-\alpha \xi} \int_{0}^{\xi} \int_{0}^{s} \frac{(s-\tau)^{q-2}}{\Gamma(q-1)} v_{*}(\tau) d \tau d s\right. \\
& \left.-\frac{1}{1-\alpha \xi} \int_{0}^{1} \frac{(1-s)^{q-1}}{\Gamma(q)} v_{*}(s) d s\right) .
\end{aligned}
$$

Let us consider the linear operator $\Theta: L^{1}([0,1], \mathbb{R}) \rightarrow C([0,1], \mathbb{R})$ given by

$$
\begin{aligned}
v \longmapsto & \Theta(v)(t) \\
= & \int_{0}^{t} \frac{(t-s)^{q-1}}{\Gamma(q)} v(s) d s \\
& +\frac{t}{1-\alpha \xi}\left(\alpha \int_{0}^{\xi} \int_{0}^{s} \frac{(s-\tau)^{q-2}}{\Gamma(q-1)} v(\tau) d \tau d s-\int_{0}^{1} \frac{(1-s)^{q-1}}{\Gamma(q)} v(s) d s\right) \\
& +\beta g\left(\int_{0}^{\eta} \frac{(\eta-s)^{q-2}}{\Gamma(q-1)} v(s) d s+\frac{\alpha}{1-\alpha \xi} \int_{0}^{\xi} \int_{0}^{s} \frac{(s-\tau)^{q-2}}{\Gamma(q-1)} v(\tau) d \tau d s\right. \\
& \left.-\frac{1}{1-\alpha \xi} \int_{0}^{1} \frac{(1-s)^{q-1}}{\Gamma(q)} v(s) d s\right) .
\end{aligned}
$$

Observe that

$$
\begin{aligned}
& \left\|h_{n}(t)-h_{*}(t)\right\| \\
& =\| \int_{0}^{t} \frac{(t-s)^{q-1}}{\Gamma(q)}\left(v_{n}(s)-v_{*}(s)\right) d s \\
& \quad+\frac{t}{1-\alpha \xi}\left(\alpha \int_{0}^{\xi} \int_{0}^{s} \frac{(s-\tau)^{q-2}}{\Gamma(q-1)}\left(v_{n}(\tau)-v_{*}(\tau)\right) d \tau d s-\int_{0}^{1} \frac{(1-s)^{q-1}}{\Gamma(q)} v(s) d s\right) \\
& \quad+\beta g\left(\int_{0}^{\eta} \frac{(\eta-s)^{q-2}}{\Gamma(q-1)}\left(v_{n}(s)-v_{*}(s)\right) d s\right. \\
& \quad+\frac{\alpha}{1-\alpha \xi} \int_{0}^{\xi} \int_{0}^{s} \frac{(s-\tau)^{q-2}}{\Gamma(q-1)}\left(v_{n}(\tau)-v_{*}(\tau)\right) d \tau d s \\
& \left.\quad-\frac{1}{1-\alpha \xi} \int_{0}^{1} \frac{(1-s)^{q-1}}{\Gamma(q)}\left(v_{n}(s)-v_{*}(s)\right) d s\right) \| \rightarrow 0
\end{aligned}
$$

as $n \rightarrow \infty$.

Thus, it follows by Lemma 2.8 that $\Theta \circ S_{F}$ is a closed graph operator. Further, we have $h_{n}(t) \in \Theta\left(S_{F, x_{n}}\right)$. Since $x_{n} \rightarrow x_{*}$, therefore we have

$$
\begin{aligned}
h_{*}(t)= & \int_{0}^{t} \frac{(t-s)^{q-1}}{\Gamma(q)} v_{*}(s) d s \\
& +\frac{t}{1-\alpha \xi}\left(\alpha \int_{0}^{\xi} \int_{0}^{s} \frac{(s-\tau)^{q-2}}{\Gamma(q-1)} v_{*}(\tau) d \tau d s-\int_{0}^{1} \frac{(1-s)^{q-1}}{\Gamma(q)} v_{*}(s) d s\right)
\end{aligned}
$$




$$
\begin{aligned}
& +\beta g\left(\int_{0}^{\eta} \frac{(\eta-s)^{q-2}}{\Gamma(q-1)} v_{*}(s) d s+\frac{\alpha}{1-\alpha \xi} \int_{0}^{\xi} \int_{0}^{s} \frac{(s-\tau)^{q-2}}{\Gamma(q-1)} v_{*}(\tau) d \tau d s\right. \\
& \left.-\frac{1}{1-\alpha \xi} \int_{0}^{1} \frac{(1-s)^{q-1}}{\Gamma(q)} v_{*}(s) d s\right)
\end{aligned}
$$

for some $v_{*} \in S_{F, x_{*}}$.

Finally, we show that there exists an open set $U \subseteq C([0,1], \mathbb{R})$ with $x \notin \Omega_{F}(x)$ for any $\lambda \in(0,1)$ and all $x \in \partial U$. Let $\lambda \in(0,1)$ and $x \in \lambda \Omega_{F}(x)$. Then there exists $v \in L^{1}([0,1], \mathbb{R})$ with $v \in S_{F, x}$ such that, for $t \in[0,1]$, we have

$$
\begin{aligned}
x(t)= & \lambda \int_{0}^{t} \frac{(t-s)^{q-1}}{\Gamma(q)} v(s) d s \\
& +\lambda \frac{t}{1-\alpha \xi}\left(\alpha \int_{0}^{\xi} \int_{0}^{s} \frac{(s-\tau)^{q-2}}{\Gamma(q-1)} v(\tau) d \tau d s-\int_{0}^{1} \frac{(1-s)^{q-1}}{\Gamma(q)} v(s) d s\right) \\
& +\lambda \beta g\left(\int_{0}^{\eta} \frac{(\eta-s)^{q-2}}{\Gamma(q-1)} v(s) d s+\frac{\alpha}{1-\alpha \xi} \int_{0}^{\xi} \int_{0}^{s} \frac{(s-\tau)^{q-2}}{\Gamma(q-1)} v(\tau) d \tau d s\right. \\
& \left.-\frac{1}{1-\alpha \xi} \int_{0}^{1} \frac{(1-s)^{q-1}}{\Gamma(q)} v(s) d s\right)
\end{aligned}
$$

Using the computations of the second step above we have

$$
\begin{aligned}
|x(t)| \leq & \psi(\|x\|)\|\phi\|\left\{\frac{1}{\Gamma(q+1)}+\frac{1}{|1-\alpha \xi|}\left(\frac{|\alpha| \xi^{q}}{\Gamma(q+1)}+\frac{1}{\Gamma(q+1)}\right)\right. \\
& \left.+|\beta|\left(\frac{\eta^{q-1}}{\Gamma(q)}+\frac{|\alpha|}{|1-\alpha \xi|} \frac{\xi^{q}}{\Gamma(q+1)}+\frac{1}{|1-\alpha \xi|} \frac{1}{\Gamma(q+1)}\right)\right\} \\
= & \psi(\|x\|)\|\phi\| \Lambda .
\end{aligned}
$$

Consequently, we have

$$
\frac{\|x\|}{\psi(\|x\|)\|\phi\| \Lambda} \leq 1 .
$$

In view of $\left(\mathrm{H}_{3}\right)$, there exists $M$ such that $\|x\| \neq M$. Let us set

$$
U=\{x \in C([0,1], \mathbb{R}):\|x\|<M\} .
$$

Note that the operator $\Omega_{F}: \bar{U} \rightarrow \mathcal{P}(C([0,1], \mathbb{R}))$ is upper semicontinuous and completely continuous. From the choice of $U$, there is no $x \in \partial U$ such that $x \in \lambda \Omega_{F}(x)$ for some $\lambda \in$ $(0,1)$. Consequently, by the nonlinear alternative of Leray-Schauder type (Lemma 2.9), we deduce that $\Omega_{F}$ has a fixed point $x \in \bar{U}$ which is a solution of problem (1.1)-(1.2). This completes the proof.

Finally, we assume that the multivalued map $F$ in (1.1) is not necessarily convex valued and formulate (and prove) the existence result as follows.

Theorem 3.4 (The lower semicontinuous case) Assume that $\left(\mathrm{A}_{3}\right),\left(\mathrm{H}_{2}\right),\left(\mathrm{H}_{3}\right)$ and the following condition hold: 
$\left(\mathrm{H}_{4}\right) \quad F:[0,1] \times \mathbb{R} \rightarrow \mathcal{P}(\mathbb{R})$ is a nonempty compact-valued multivalued map such that

(a) $(t, x) \longmapsto F(t, x)$ is $\mathcal{L} \otimes \mathcal{B}$ measurable,

(b) $x \longmapsto F(t, x)$ is lower semicontinuous for each $t \in[0,1]$;

then the boundary value problem (1.1)-(1.2) has at least one solution on $[0,1]$.

Proof It follows from $\left(\mathrm{H}_{2}\right)$ and $\left(\mathrm{H}_{4}\right)$ that $F$ is of l.s.c. type. Then, from Lemma 2.12, there exists a continuous function $f: C^{2}([0,1], \mathbb{R}) \rightarrow L^{1}([0,1], \mathbb{R})$ such that $f(x) \in \mathcal{F}(x)$ for all $x \in C([0,1], \mathbb{R})$.

Consider the problem

$$
\left\{\begin{array}{l}
{ }^{c} D^{q} x(t)=f(x(t)), \quad t \in[0,1], 1<q \leq 2, \\
x(0)=\beta g\left(x^{\prime}(\eta)\right), \quad x^{\prime}(1)=\alpha \int_{0}^{\xi} x^{\prime}(s) d s, \quad 0<\xi, \eta<1 .
\end{array}\right.
$$

Observe that if $x \in C^{2}([0,1], \mathbb{R})$ is a solution of (3.3), then $x$ is a solution to problem (1.1)-(1.2). In order to transform problem (3.3) into a fixed point problem, we define the operator $\overline{\Omega_{F}}$ as

$$
\begin{aligned}
\overline{\Omega_{F}} x(t)= & \int_{0}^{t} \frac{(t-s)^{q-1}}{\Gamma(q)} f(x(s)) d s \\
& +\frac{t}{1-\alpha \xi}\left(\alpha \int_{0}^{\xi} \int_{0}^{s} \frac{(s-\tau)^{q-2}}{\Gamma(q-1)} f(x(\tau)) d \tau d s-\int_{0}^{1} \frac{(1-s)^{q-1}}{\Gamma(q)} f(x(s)) d s\right) \\
& +\beta g\left(\int_{0}^{\eta} \frac{(\eta-s)^{q-2}}{\Gamma(q-1)} f(x(s)) d s+\frac{\alpha}{1-\alpha \xi} \int_{0}^{\xi} \int_{0}^{s} \frac{(s-\tau)^{q-2}}{\Gamma(q-1)} f(x(\tau)) d \tau d s\right. \\
& \left.-\frac{1}{1-\alpha \xi} \int_{0}^{1} \frac{(1-s)^{q-1}}{\Gamma(q)} f(x(s)) d s\right), \quad t \in[0,1] .
\end{aligned}
$$

It can easily be shown that $\overline{\Omega_{F}}$ is continuous and completely continuous. The remaining part of the proof is similar to that of Theorem 3.3. So we omit it. This completes the proof.

\section{Examples}

Consider the problem

$$
\begin{cases}{ }^{c} D^{3 / 2} x(t) \in F(t, x(t)), & 0 \leq t \leq 1 \\ x(0)=\frac{1}{3} g\left(x^{\prime}(3 / 4)\right), & x^{\prime}(1)=\frac{1}{2} \int_{0}^{1 / 3} x^{\prime}(s) d s .\end{cases}
$$

Here, $q=3 / 2, \alpha=1 / 2, \xi=1 / 3, \beta=1 / 3, \eta=3 / 4$ and

$$
g(v)= \begin{cases}\sqrt{v}, & |v| \geq 1 \\ v^{2}, & |v|<1\end{cases}
$$

With the given values, we find that $\Lambda \approx 2.3980168$.

(i) Consider the multivalued map $F:[0,1] \times \mathbb{R} \rightarrow \mathcal{P}(\mathbb{R})$ given by

$$
F(t, x)=\left[0, \frac{1}{6}(t+1) \sin x+\frac{1}{6}\right]
$$


Then we have

$$
\sup \{|v|: v \in F(t, x)\} \leq \frac{1}{6}(t+1)+\frac{1}{6}
$$

and

$$
H_{d}(F(t, x), F(t, \bar{x})) \leq \frac{1}{6}(t+1)|x-\bar{x}| .
$$

Let $m(t)=\frac{1}{6}(t+1)$. Then $\|m\|=\frac{1}{3}$ and $\|m\| \Lambda \approx 0.7993389<1$. Hence by Theorem 3.2 problem (4.1) has a solution.

(ii) Let $F:[0,1] \times \mathbb{R} \rightarrow \mathcal{P}(\mathbb{R})$ be a multivalued map given by

$$
F(t, x)=\left[e^{-t}\left(\frac{|x|^{3}}{|x|^{3}+5}+9\right), 2 e^{-t}\left(\frac{|x|^{3}}{|x|^{3}+3}+1\right)\right] .
$$

For $v \in F$, we have

$$
|v(t)| \leq \max \left(e^{-t}\left(\frac{|x|^{3}}{|x|^{3}+5}+9\right), 2 e^{-t}\left(\frac{|x|^{3}}{|x|^{3}+3}+1\right)\right) \leq 10 e^{-t}, \quad x \in \mathbb{R} .
$$

Thus

$$
\|F(t, x)\|_{\mathcal{P}}:=\sup \{|y|: y \in F(t, x)\} \leq 10 e^{-t}=\phi(t) \psi(\|x\|),
$$

with $\phi(t)=e^{-t}, \psi(\|x\|)=10$.

By assumption $\left(\mathrm{H}_{3}\right)$, we find that $M>23.980168$. It follows by Theorem 3.3 that problem (4.1) has a solution.

\section{Existence results for problem (1.1)-(1.3)}

To define the solution for problem (1.1)-(1.3), we need the following lemma.

Lemma 5.1 Let $y \in C([0,1], \mathbb{R})$ and $x \in C^{2}([0,1], \mathbb{R})$ be a solution of the linear boundary value problem

$$
\left\{\begin{array}{l}
{ }^{c} D^{q} x(t)=y(t), \quad t \in[0,1], 1<q \leq 2, \\
x^{\prime}(0)=\alpha \int_{0}^{\xi} x^{\prime}(s) d s, \quad x(1)=\beta g\left(x^{\prime}(\eta)\right), \quad 0 \leq \xi, \eta \leq 1 .
\end{array}\right.
$$

Then

$$
\begin{aligned}
x(t)= & \int_{0}^{t} \frac{(t-s)^{q-1}}{\Gamma(q)} y(s) d s \\
& +(t-1) \frac{\alpha}{1-\alpha \xi} \int_{0}^{\xi} \int_{0}^{s} \frac{(s-\tau)^{q-2}}{\Gamma(q-1)} y(\tau) d \tau d s-\int_{0}^{1} \frac{(1-s)^{q-1}}{\Gamma(q)} y(s) d s \\
& +\beta g\left(\int_{0}^{\eta} \frac{(\eta-s)^{q-2}}{\Gamma(q-1)} y(s) d s+\frac{\alpha}{1-\alpha \xi} \int_{0}^{\xi} \int_{0}^{s} \frac{(s-\tau)^{q-2}}{\Gamma(q-1)} y(\tau) d \tau d s\right) .
\end{aligned}
$$

Proof We omit the proof as it is similar to that of Lemma 2.3. 
In relation to problem (1.1)-(1.3), we define an operator $G_{F}: C([0,1], \mathbb{R}) \rightarrow \mathcal{P}(C([0,1], \mathbb{R}))$ by

$$
G_{F}(x)=\left\{\begin{array}{l}
h \in C([0,1], \mathbb{R}): \\
h(t)=\left\{\begin{array}{c}
\int_{0}^{t} \frac{(t-s)^{q-1}}{\Gamma(q)} v(s) d s \\
\quad+(t-1) \frac{\alpha}{1-\alpha \xi} \int_{0}^{\xi} \int_{0}^{s} \frac{(s-\tau)^{q-2}}{\Gamma(q-1)} v(\tau) d \tau d s-\int_{0}^{1} \frac{(1-s)^{q-1}}{\Gamma(q)} v(s) d s \\
+\beta g\left(\int_{0}^{\eta} \frac{\left(\eta-s q^{q-2}\right.}{\Gamma(q-1)} v(s) d s+\frac{\alpha}{1-\alpha \xi} \int_{0}^{\xi} \int_{0}^{s} \frac{(s-\tau) q^{q-2}}{\Gamma(q-1)} v(\tau) d \tau d s\right)
\end{array}\right\}
\end{array}\right.
$$

for $v \in S_{F, x}$, and set

$$
\bar{\Lambda}=\frac{1}{\Gamma(q+1)}\left(2+q|\beta| \eta^{q-1}+\frac{|\alpha|(1+|\beta|) \xi^{q}}{|1-\alpha \xi|}\right) .
$$

With the above operator and estimate (5.3), we can reproduce all the existence results obtained in Section 3 for the boundary value problem (1.1)-(1.3).

\section{Conclusions}

We have studied the existence of solutions for fractional-order differential inclusions supplemented with new nonlocal nonlinear flux type integral boundary conditions for different types of multivalued maps involved in the given problems. Precisely, Section 3 contains some existence results for problem (1.1)-(1.2): the first one (Theorem 3.2) deals with nonconvex valued maps (the Lipschitz case) and is obtained by applying a fixed point theorem for multivalued maps due to Covitz and Nadler; the second result (Theorem 3.3) takes into account the convex-valued maps (the upper semicontinuous case) and relies on nonlinear alternative of Leray-Schauder type; and the third one (Theorem 3.4) involves multivalued maps which are not necessarily convex-valued (the lower semicontinuous case) and is obtained by jointly using the nonlinear alternative of Leray-Schauder type and the selection theorem of Bressan and Colombo [37] for lower semicontinuous maps with decomposable values. These results have been illustrated with the aid of examples in Section 4. Finally, we have provided the platform for proving the existence results for problem (1.1)-(1.3). We emphasize that our results are new, and several special results can be obtained by fixing the parameters $\alpha, \beta, \xi, \eta$ involved in the problems at hand.

\section{Competing interests}

The authors declare that they have no competing interests.

\section{Authors' contributions}

Each of the authors, BA, SKN, AA and FA contributed to each part of this work equally and read and approved the final version of the manuscript.

\section{Author details}

${ }^{1}$ Nonlinear Analysis and Applied Mathematics (NAAM)-Research Group, Department of Mathematics, Faculty of Science, King Abdulaziz University, P.O. Box 80203, Jeddah, 21589, Saudi Arabia. ${ }^{2}$ Department of Mathematics, University of loannina, loannina, 451 10, Greece.

\section{Acknowledgements}

This article was funded by the Deanship of Scientific Research (DSR), King Abdulaziz University. The authors, therefore, acknowledge with thanks DSR technical and financial support. The authors also acknowledge the reviewers for their useful comments. 


\section{References}

1. Podlubny, I: Fractional Differential Equations. Academic Press, San Diego (1999)

2. Kilbas, AA, Srivastava, HM, Trujillo, JJ: Theory and Applications of Fractional Differential Equations. North-Holland Mathematics Studies, vol. 204. Elsevier, Amsterdam (2006)

3. Sabatier, J, Agrawal, OP, Machado, JAT (eds.): Advances in Fractional Calculus: Theoretical Developments and Applications in Physics and Engineering. Springer, Dordrecht (2007)

4. Tomovski, Z, Hilfer, R, Srivastava, HM: Fractional and operational calculus with generalized fractional derivative operators and Mittag-Leffler type functions. Integral Transforms Spec. Funct. 21, 797-814 (2010)

5. Konjik, S, Oparnica, L, Zorica, D: Waves in viscoelastic media described by a linear fractional model. Integral Transforms Spec. Funct. 22, 283-291 (2011)

6. Keyantuo, V, Lizama, C: A characterization of periodic solutions for time-fractional differential equations in UMD spaces and applications. Math. Nachr. 284, 494-506 (2011)

7. Ahmad, B, Nieto, JJ: Riemann-Liouville fractional integro-differential equations with fractional nonlocal integral boundary conditions. Bound. Value Probl. 2011, Article ID 36 (2011)

8. Liang, S, Zhang, J: Existence of multiple positive solutions for $m$-point fractional boundary value problems on an infinite interval. Math. Comput. Model. 54, 1334-1346 (2011)

9. Su, X: Solutions to boundary value problem of fractional order on unbounded domains in a Banach space. Nonlinear Anal. 74, 2844-2852 (2011)

10. Bai, ZB, Sun, W: Existence and multiplicity of positive solutions for singular fractional boundary value problems. Comput. Math. Appl. 63, 1369-1381 (2012)

11. Agarwal, RP, O'Regan, D, Stanek, S: Positive solutions for mixed problems of singular fractional differential equations. Math. Nachr. 285, 27-41 (2012)

12. Cabada, A, Wang, G: Positive solutions of nonlinear fractional differential equations with integral boundary value conditions. J. Math. Anal. Appl. 389, 403-411 (2012)

13. Ahmad, B, Ntouyas, SK, Alsaedi, A: A study of nonlinear fractional differential equations of arbitrary order with Riemann-Liouville type multistrip boundary conditions. Math. Probl. Eng. 2013, Article ID 320415 (2013)

14. O'Regan, D, Stanek, S: Fractional boundary value problems with singularities in space variables. Nonlinear Dyn. 71 641-652(2013)

15. Graef, JR, Kong, L, Wang, M: Existence and uniqueness of solutions for a fractional boundary value problem on a graph. Fract. Calc. Appl. Anal. 17, 499-510 (2014)

16. Wang, G, Liu, S, Zhang, L: Eigenvalue problem for nonlinear fractional differential equations with integral boundary conditions. Abstr. Appl. Anal. 2014, Article ID 916260 (2014)

17. Ahmad, B, Nieto, JJ: Sequential differential equations of fractional order with multi-point boundary conditions. Georgian Math. J. 21, 243-248 (2014)

18. Ahmad, B, Agarwal, RP: Some new versions of fractional boundary value problems with slit-strips conditions. Bound Value Probl. 2014, Article ID 175 (2014)

19. Smirnov, GV: Introduction to the Theory of Differential Inclusions. Graduate Studies in Mathematics, vol. 41. Am. Math. Soc., Providence (2002)

20. Chang, Y-K, Li, WT, Nieto, JJ: Controllability of evolution differential inclusions in Banach spaces. Nonlinear Anal. 67 , 623-632 (2007)

21. Li, WS, Chang, YK, Nieto, JJ: Solvability of impulsive neutral evolution differential inclusions with state-dependent delay. Math. Comput. Model. 49, 1920-1927 (2009)

22. Henderson, J, Ouahab, A: Fractional functional differential inclusions with finite delay. Nonlinear Anal. 70, 2091-2105 (2009)

23. Ahmad, B, Nieto, JJ, Pimentel, J: Some boundary value problems of fractional differential equations and inclusions. Comput. Math. Appl. 62, 1238-1250 (2011)

24. Ahmad, B, Ntouyas, SK: Existence results for nonlocal boundary value problems of fractional differential equations and inclusions with strip conditions. Bound. Value Probl. 2012, Article ID 55 (2012)

25. Ahmad, B, Ntouyas, SK: Existence results for higher order fractional differential inclusions with multi-strip fractional integral boundary conditions. Electron. J. Qual. Theory Differ. Equ. 2013, 20 (2013)

26. Dhage, BC, Ntouyas, SK: Existence results for boundary value problems for fractional hybrid differential inclusions. Topol. Methods Nonlinear Anal. 44, 229-238 (2014)

27. Agarwal, RP, Baleanu, D, Hedayati, V, Rezapour, S: Two fractional derivative inclusion problems via integral boundary condition. Appl. Math. Comput. 257, 205-212 (2015)

28. Cernea, A: Filippov lemma for a class of Hadamard-type fractional differential inclusions. Fract. Calc. Appl. Anal. 18 163-171 (2015)

29. Deimling, K: Multivalued Differential Equations. de Gruyter, Berlin (1992)

30. Hu, S, Papageorgiou, N: Handbook of Multivalued Analysis, Theory I. Kluwer Academic, Dordrecht (1997)

31. Kisielewicz, M: Differential Inclusions and Optimal Control. Kluwer Academic, Dordrecht (1991)

32. Covitz, H, Nadler, SB Jr.: Multivalued contraction mappings in generalized metric spaces. Isr. J. Math. 8, 5-11 (1970)

33. Lasota, A, Opial, Z: An application of the Kakutani-Ky Fan theorem in the theory of ordinary differential equations. Bull. Acad. Pol. Sci., Sér. Sci. Math. Astron. Phys. 13, 781-786 (1965)

34. Granas, A, Dugundji, J: Fixed Point Theory. Springer, New York (2005)

35. Frigon, M: Théorèmes d'existence de solutions d'inclusions différentielles. In: Granas, A, Frigon, M (eds.) Topological Methods in Differential Equations and Inclusions. NATO ASI Series C, vol. 472, pp. 51-87. Kluwer Academic, Dordrecht (1995)

36. Castaing, C, Valadier, M: Convex Analysis and Measurable Multifunctions. Lecture Notes in Mathematics, vol. 580. Springer, Berlin (1977)

37. Bressan, A, Colombo, G: Extensions and selections of maps with decomposable values. Stud. Math. 90, 69-86 (1988) 